\title{
SUPPURATIVE OTITIS MEDIA IN CHILDREN IN ANGOLA
}

\section{$\underline{\text { M. Filipe }}{ }^{1}$, Å. Reimer², F. Uddén ${ }^{3}$, T. Pelkonen ${ }^{4}$, K. Riesbeck ${ }^{3}$.}

${ }^{1}$ Department of ENT,Faculty of Medicine, University Agosthino Neto, Luanda, Angola.

${ }^{2}$ Näshälsan Höllviken AB, Höllviken, Sweden.

${ }^{3}$ Clinical Microbiology, Dept. of Translational Medicine, Faculty of Medicine, Lund University, Malmö, Sweden.

${ }^{4}$ Helsinki University Hospital, Children's Hospital, Helsinki, Finland and University of Helsinki, Helsinki, Finland.

Background: Chronic suppurative otitis media (CSOM) is an important cause of hearing loss in children and constitutes a serious health problem globally. We studied a cohort of children with otorrhoea in five provinces of Angola, a Sub-Saharan country.

Methods : Children attending services of oto-rhino-laryngology due to otorrhoea were submitted to full clinical examination and interview. Ear secretion and nasopharyngeal samples were collected, transported frozen, and cultured in Sweden using standard techniques

Results : Unilateral disease was detected in $76 \%$, bilateral in $24 \%$ of children. Otorrhoea had lasted $<15$ days in $24 \%$, and $\geq 1$ year in $39 \%$. Clinical hearing loss presented in $49 \%$ of children ( 96 of 196), and correlated with the duration of otorrhoea $(p=0.003$ ). When comparing with the capital province Luanda, a poorer Lunda sul province, otorrhoea had lasted longer and hearing loss was more common $(p=0.02)$. Ear discharge specimens $(n=133)$ and nasopharyngeal swabs $(n=108)$ yielded 376 and 212 individual isolates, respectively. Proteus spp. (13.3\%), Pseudomonas aeruginosa (10.4\%) and Enterococcus spp. (10.4\%) were dominating in ear discharge. Pneumococci and Staphylococcus aureus were detected in $15.1 \%$ and $7.1 \%$ of nasopharyngeal samples, respectively. Resistance rate to quinolones exceeded 10\% among Enterobacteriaceae and was 30.8\% in Staphylococcus aureus, whereas 6.3\% of P. aeruginosa was resistant.

\section{Conclusions}

- In Angola, otorrhoea is long-lasting, causes hearing loss, and associates with resource-limited living conditions.

- Bacterial cultures of ear discharge reveal difficult-to-treat Gram-negative bacteria.

- Ear infections should be treated early to avoid severe sequelae. 